\title{
Letter
}

Dermatology

Dermatology 2021;237:145-147

DOI: $10.1159 / 000504408$

\section{Prominent Transepidermal Melanin Deposition Is a Distinguishing Histopathological Feature of Melasma: A Clinicopathologic Study}

\author{
Farhaan Hafeez ${ }^{\mathrm{a}, \mathrm{b}}$ Douglas A. Mata ${ }^{\mathrm{a}}$ Christine G. Lian ${ }^{\mathrm{a}}$ \\ Evangelos G. Poulos ${ }^{\mathrm{c}}$ \\ a Department of Pathology, Brigham and Women's Hospital, \\ Boston, MA, USA; ${ }^{b}$ Department of Dermatology, St. Luke's \\ University Health System, Temple School of Medicine, \\ Bethlehem, PA, USA; ${ }^{\circ}$ Department of Dermatology and \\ Cutaneous Surgery, University of Miami Miller School of \\ Medicine, Miami, FL, USA
}

\section{Keywords}

Melasma · Postinflammatory hyperpigmentation ·

Disorders of hyperpigmentation - Clinicopathologic study .

Dermatopathology

\section{Dear Editor,}

Melasma and postinflammatory hyperpigmentation (PIH) are the most common causes of acquired hyperpigmentation. Melasma has been described as having increased melanin in all layers of the epidermis [1-3], a "cap" of melanin pigment located over keratinocytic nuclei $[2,4]$, variable changes in melanocyte density, and an increase in dermal melanophages, mast cells, and solar elastosis $[2,4,5]$. PIH has been described as having variable changes in basal layer melanin deposition, variable changes at the dermalepidermal junction, and an increase in dermal melanophages [3, 4]. Clinical presentation and history are the most important means of distinguishing melasma and $\mathrm{PIH}$; however, these diagnoses are occasionally difficult to distinguish clinically, and validated histopathological criteria to differentiate between melasma and PIH in the literature are lacking. The purpose of this study was to histologically compare cases of melasma and PIH. Of the previously described histological attributes of melasma, we hypothesized that prominent melanin deposition in all layers of the epidermis, i.e., transepidermal melanin deposition, as detected by Fontana-Masson staining, would favor a diagnosis of melasma over PIH.

Cases of melasma and PIH diagnosed between July 4, 2010 and July 23, 2018 were sought from the pathology archives of Aurora Diagnostics Global Pathology (Miami Lakes, FL, USA) and the Brigham and Women's Hospital (Boston, MA, USA). Medical records, the site of biopsy, and/or clinical photographs were evaluated by a dermatologist (F.H.) to ensure the accuracy of the diagnosis. Cases with clinical presentations that were inconsistent with the histological diagnosis were excluded. In total, 30 biopsies (27 patients) of melasma and 37 biopsies (35 patients) of PIH were obtained and compared using the histopathological criteria listed
Fig. 1. Representative photomicrographs of melasma and postinflammatory hyperpigmentation. a PIH. H\&E. b PIH. FM. c Melasma. H\&E. d Melasma. FM. All pictures were taken at $200 \times$. Prominent transepidermal melanin deposition is noted in the melasma sample but is absent in the $\mathrm{PIH}$ sample. PIH, postinflammatory hyperpigmentation. H\&E, hematoxylin and eosin. FM, Fontana-Masson.
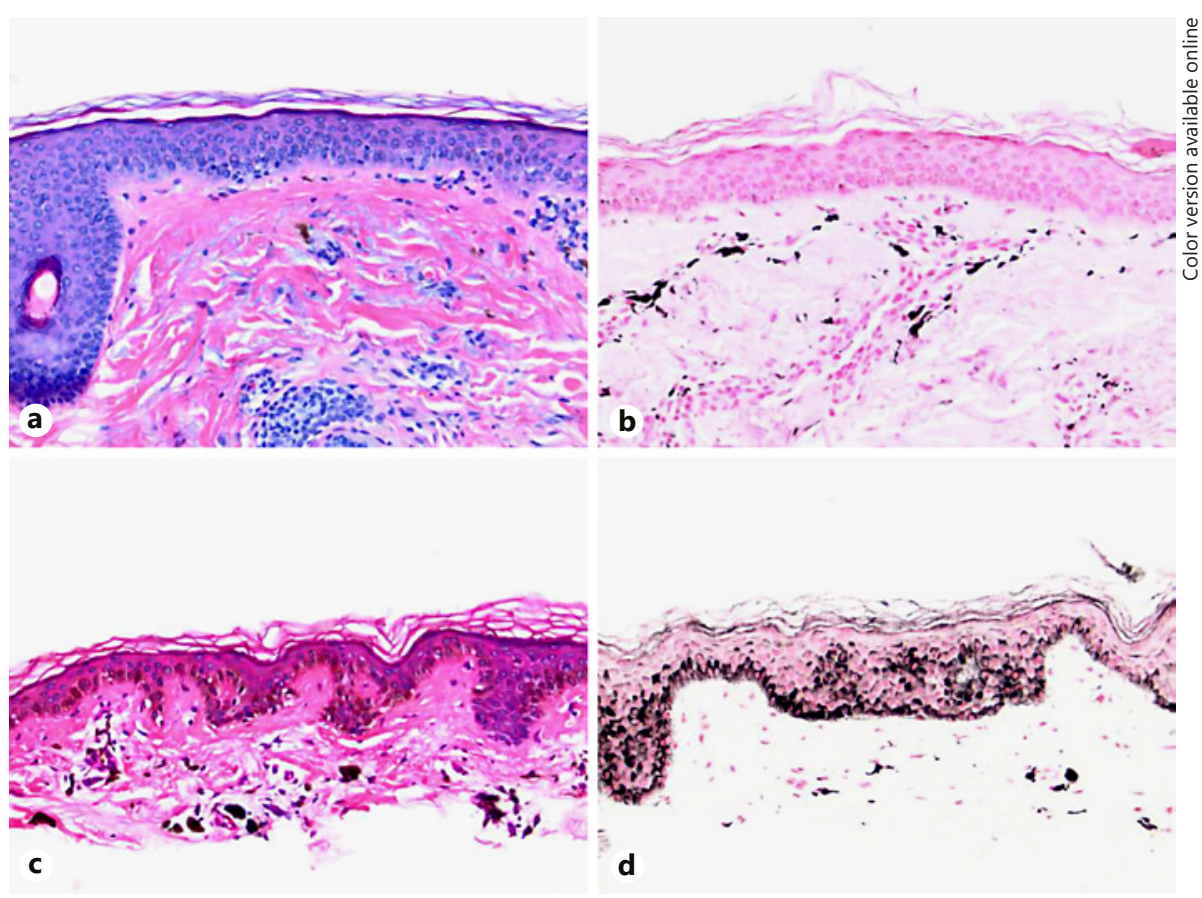

karger@karger.com

www.karger.com/drm

(c) 2019 S. Karger AG, Basel

Karger
Farhaan Hafeez

Department of Pathology, Brigham and Women's Hospital

Harvard Medical School, 75 Francis Street

Boston, MA 02115 (USA)

E-Mail farhaanhafeez@gmail.com 
Table 1. Histologic comparison between melasma and postinflammatory hyperpigmentation (PIH)

\begin{tabular}{|c|c|c|c|c|c|c|c|}
\hline & $\begin{array}{l}\text { Melasma } \\
(n=30)\end{array}$ & $\begin{array}{l}\text { PIH } \\
(n=37)\end{array}$ & $p$ value & & $\begin{array}{l}\text { Melasma } \\
(n=30)\end{array}$ & $\begin{array}{l}\text { PIH } \\
(n=37)\end{array}$ & $p$ value \\
\hline Age, years & & & & \multicolumn{4}{|l|}{ Solar elastosis } \\
\hline Sex & & & & 1 & 10 & 6 & \\
\hline Male & 7 & 3 & 0.06 & 2 & 0 & 1 & \\
\hline Female & 11 & 23 & & Not specified/undetermined & 13 & 11 & \\
\hline Sun-exposed skin & 17 & 24 & 0.04 & 0 & 5 & 6 & 0.72 \\
\hline Unexposed skin & 1 & 12 & & 1 & 7 & 13 & \\
\hline Not specified/undetermined & 12 & 1 & & 2 & 4 & 7 & \\
\hline Basket weave stratum corneum & & & & Not specified/undetermined & 14 & 11 & \\
\hline Present & 28 & 34 & 1.00 & Average score $(0-2) \pm \mathrm{SD}$ & $0.9 \pm 0.8$ & $1.0 \pm 0.7$ & \\
\hline Absent & 28 & 34 & & Not specified/undetermined & 23 & 11 & \\
\hline Not specified/undetermined & 0 & 0 & & Average score $(0-2) \pm \mathrm{SD}$ & $1.0 \pm 0$ & $1.3 \pm 0.5$ & \\
\hline Rete ridge effacement & & & & Mast cells (IHC with CD117) & & & \\
\hline Present & 4 & 10 & 0.36 & 0 & 5 & 25 & 0.35 \\
\hline Absent & 22 & 27 & & 1 & 1 & 1 & \\
\hline Not specified/undetermined & 4 & 0 & & 2 & 0 & 0 & \\
\hline Dermal melanophages (FM) & & & & Not specified/undetermined & 24 & 11 & \\
\hline Present & 20 & 31 & 0.36 & Average score $(0-2) \pm \mathrm{SD}$ & $0.2 \pm 0.4$ & $0.04 \pm 0.2$ & \\
\hline
\end{tabular}

Absent

Not specified/undetermined $2 \quad 0$

Melanin cap (FM)

Present $21 \quad 20$

Absent $\quad 7 \quad 14$

Not specified/undetermined 23

Transepidermal melanin deposition ${ }^{\mathrm{a}}$ (FM)

\begin{tabular}{lccc}
0 & 2 & 15 & $3.6 \times 10^{-6}$ \\
1 & 5 & 14 & \\
2 & 21 & 5 & \\
Not specified/undetermined & 2 & 3 & \\
Average score $(0-2) \pm \mathrm{SD}$ & $1.7 \pm 0.6$ & $0.7 \pm 0.7$ \\
\hline
\end{tabular}

in Table 1. Differences among variables were assessed using Fisher's exact test. Statistical tests were two-sided and used a significance threshold of $p<0.05$.

As shown in Table 1, cases of melasma demonstrated a significantly greater degree of transepidermal melanin deposition $(p=$ $\left.3.6 \times 10^{-6}\right)$ and solar elastosis $(p=0.03)$ compared to cases of PIH on univariate analysis. After simultaneously adjusting for both biopsy site and skin phototype, an increase in transepidermal melanin deposition was still associated with the diagnosis of melasma (rather than $\mathrm{PIH}$ ) on multivariate logistic regression analysis $(p=$ $0.02)$. However, solar elastosis was no longer associated with melasma (rather than PIH) after controlling for these variables ( $p=$ $0.20)$. Representative photomicrographs are shown in Figure 1.
H\&E, hematoxylin and eosin. FM, Fontana-Masson. IHC, immunohistochemistry. SD, standard deviation. For the variables categorized as either 0,1 , or 2: $0=$ none to minimally increased/ normal, 1 = moderately increased, and $2=$ markedly increased.

${ }^{a}$ For transepidermal melanin deposition, a score of 0 was given for no epidermal melanin or melanin localized only to basal layer, a score of 1 for melanin deposition present in suprabasal layers but only focally, and a score of 2 for multifocal presence of suprabasal melanin deposition.

${ }^{\mathrm{b}}$ Melanocyte density was determined using SOX10 immunohistochemistry and reflects the ratio of epidermal melanocytes to basal keratinocytes (MC/KC). $0=\mathrm{MC} / \mathrm{KC}<0.05,1=0.05 \leq \mathrm{MC} /$ $\mathrm{KC} \leq 0.15,2=\mathrm{MC} / \mathrm{KC}>0.15$.

Melasma has been hypothesized to be due to hyperfunctional melanocytes activated by a trigger (e.g., UV irradiation) [1], and prominent transepidermal melanin deposition may represent the histologic correlate of this melanocytic hyperactivity. Other attributes that have been associated with melasma and PIH were not distinguishing features in this study [2-5].

The limitations of this study merit consideration. Given that melasma and PIH are essentially clinical diagnoses that require clinicopathological correlation in challenging circumstances, the final diagnoses were rendered only after reviewing histology as well as all available clinical information, including physical examination, clinical notes, and/or photographs. 
Many of the histologic features attributed to diseases of hyperpigmentation reported in the literature are not unique and are shared by many disease entities [3, 4]. However, this study has shown that prominent transepidermal melanin deposition as detected by the Fontana-Masson stain - an accessible and inexpensive stain available in most laboratories - is a specific histologic feature of melasma and can support its diagnosis in the appropriate clinical context.

Key Message

Prominent transepidermal melanin deposition is a distinguishing feature of melasma compared to postinflammatory hyperpigmentation.

Statement of Ethics

This study was approved by the Institutional Review Board of the Brigham and Women's Hospital (Boston, MA).

\section{Disclosure Statement}

The authors have no conflicts of interest to declare.

Funding Sources

There was no funding received for this study.

\section{Author Contributions}

Farhaan Hafeez designed and executed the study and was principally responsible for writing the manuscript. Douglas A. Mata conducted the statistical analysis and revised the manuscript for important intellectual content. Christine G. Lian and Evangelos G. Poulos assisted in the design and execution of the study.

\section{References}

1 Grimes PE, Yamada N, Bhawan J. Light microscopic, immunohistochemical, and ultrastructural alterations in patients with melasma. Am J Dermatopathol. 2005 Apr;27(2):96-101.

2 Kang WH, Yoon KH, Lee ES, Kim J, Lee KB, Yim H, et al. Melasma: histopathological characteristics in 56 Korean patients. Br J Dermatol. 2002 Feb;146(2):228-37.

3 Calonje JE, Brenn T, Alexander Lazar A, McKee P. McKee's pathology of the skin. 4th ed. Philadelphia (PA): Saunders Elsevier; 2011.

4 Patterson JW. Weedon's skin pathology. 4th ed. London, UK: Churchill Livingstone; 2016.

5 Hernández-Barrera R, Torres-Alvarez B, Castanedo-Cazares JP, OrosOvalle C, Moncada B. Solar elastosis and presence of mast cells as key features in the pathogenesis of melasma. Clin Exp Dermatol. 2008 May; 33(3):305-8. 